\title{
Control of a Segway with unknown control coefficient and input constraint
}

Bong Seok Park

Division of Electrical, Electronic, and Control Engineering, Kongju National University, Cheonan, Korea

\section{]jfis}

Received: May 26, 2016

Revised : Jun. 8, 2016

Accepted: Jun. 8, 2016

Correspondence to: Bong Seok Park (bspark@kongju.ac.kr)

(c)The Korean Institute of Intelligent Systems

(c) This is an Open Access article distributed under the terms of the Creative Commons Attribution Non-Commercial License (http://creativecommons.org/licenses/ by-nc/3.0// which permits unrestricted noncommercial use, distribution, and reproduction in any medium, provided the original work is properly cited.

\begin{abstract}
This paper proposes a control method of the Segway with unknown control coefficient and input saturation. To design a simple controller for the Segway with the model uncertainty, the prescribed performance function is used. Furthermore, an auxiliary variable is introduced to deal with unknown time-varying control coefficient and input saturation problem. Due to the auxiliary variable, function approximators are not used in this paper although all model uncertainties are unknown. Thus, the controller can be simple. From the Lyapunov stability theory, it is proved that all errors of the proposed control system remain within the prescribed performance bounds. Finally, the simulation results are presented to demonstrate the performance of the proposed scheme.
\end{abstract}

Keywords: Segway, prescribed performance function, input saturation, unknown control coefficient, model uncertainty.

\section{Introduction}

Various forms of the Segway have recently attracted attention as a means of personal transportation. The reason is that it is easy to carry. Since the Segway is a personal vehicle, the safety of the passenger is very important and thus, it requires the design of a reliable controller. For this, it is necessary to consider the model uncertainty and input constraint. The weight and center of gravity of the passenger are different, and the control coefficient is time-varying because it is dependent on the angle of the inclination. These lead the model uncertainty. Furthermore, the Segway is driven by DC motor and it has a limit in the input voltage physically. Thus, we need to consider the input saturation problem for real applications.

To deal with the model uncertainty, previous researches used various methods such as neural network [1, 2], fuzzy logic [3, 4], and adaptive control technique [5, 6]. Aforementioned papers are useful to compensate the model uncertainty, but it has the disadvantage due to the complexity of the controller. For the unknown control direction, the Nussbaum gain technique [7, 8] was generally used. However, it can be unstable because it is constructed by the means of so-called amplitude elongation. Therefore, designing the controller based on the Nussbaum gain technique causes many difficulties in the physical point of view. The prescribed performance function based controller [9, 10] was recently proposed to solve the disadvantage of various methods dealing with the model uncertainty. In this approach, the error signals remain within the prescribed performance bounds without estimating the model uncertainty. Thus, it makes the controller simple. However, most of the researches using the prescribed performance function did not consider the time-varying control coefficient. 


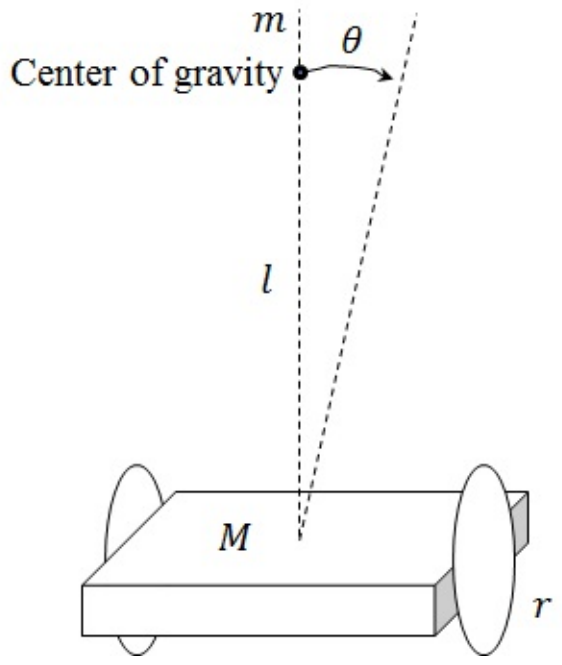

Figure 1. Segway.

Motivated by above analysis, a simple controller for the Segway with model uncertainties and input constraint is proposed to solve above problems. First, the prescribed performance function is used to deal with the model uncertainty. This means that function approximators are not used in this paper although all model uncertainties are unknown. Therefore, the controller is simple in the presence of the model uncertainty. Second, an auxiliary variable is introduced to deal with the input saturation problem and unknown time-varying control coefficient. Since the error signals of the proposed control scheme remain within the prescribed performance bounds, the safety of the passenger is guaranteed. Furthermore, the transient and steady-state responses can be adjusted by selecting the prescribed performance functions properly. Finally, the controller is designed using the Lyapunov stability theory and the simulation results are presented to verify the performance of the proposed control scheme.

\section{Segway Model}

In this paper, the Segway shown in Figure 1 is considered. The dynamic model of the Segway is as follows [11].

$$
\ddot{\theta}=(m g l \sin \theta) / I-(m F l \cos \theta) /(M+m) I,
$$

where $m$ is the mass of the passenger, $M$ is the mass of the Segway base, $I$ is the inertia of the Segway with the passenger, $\theta$ is the angle of the inclination, $g$ is the gravitational acceleration, $l$ is the length between the center of gravity of the passenger and Segway base, and $F$ is the force applied to the Segway.
Since the Segway is driven by DC motor, it is necessary to consider the motor dynamics. Neglecting the motor inductance, we have

$$
F=\frac{2 k_{t} n u}{R r}-\frac{2 k_{t} n e_{b}}{R r},
$$

where $k_{t}$ is the motor torque constant, $e_{b}$ is the back-emf voltage, $R$ is the resistance, $r$ is the radius of the wheel, $n$ is the gear reduction, $\dot{x}$ is the angular velocity of the wheel, and $u$ is the motor input voltage. Let's define $x_{1}=\theta$ and $x_{2}=\dot{\theta}$. Then, substituting (2) into (1) yields

$$
\begin{aligned}
& \dot{x}_{1}=x_{2} \\
& \dot{x}_{2}=f\left(x_{1}\right)+b\left(x_{1}\right) \bar{u}+d(t),
\end{aligned}
$$

where

$$
\begin{aligned}
& f\left(x_{1}\right)=\frac{m g l \sin x_{1}}{I}, \\
& b\left(x_{1}\right)=-\frac{2 k_{t} n m l \cos x_{1}}{(M+m) I R r}, \\
& d(t)=\frac{2 k_{t} n e_{b} m l \cos x_{1}}{(M+m) I R r} .
\end{aligned}
$$

In (3), $m$ and $l$ are dependent on the passenger. Thus, these are model uncertainties and they lead the unknown time-varying control coefficient $b\left(x_{1}\right)$. In these expressions, $\bar{u}$ is the saturated input voltage as follows.

$$
\bar{u}= \begin{cases}u_{M}, & \text { if } u \geq u_{M}, \\ u, & \text { if }-u_{M}<u<u_{M}, \\ -u_{M}, & \text { if } u \leq u_{M},\end{cases}
$$

where $u_{M}$ is the limit of the motor input voltage.

Assumption 1. In (3), the model parameters are unknown constants, but bounded. Moreover, the back-emf voltage $e_{b}$ is the unknown time-varying parameter, but bounded.

Remark 1. To deal with the unknown time-varying coefficient $b\left(x_{1}\right)$, the auxiliary variable is used. Thus, the assumption on sign of $b\left(x_{1}\right)$ is not required in this paper.

The control objective is to design the controller so that the angle of the inclination tracks the desired angle(i.e., $x_{d}=0^{\circ}$ ) in the presence of the model uncertainty and input constraint. 


\section{Controller Design}

In this section, the controller is designed using the prescribed performance functions. Define the error signals

$$
\begin{aligned}
& e_{1}=\Gamma\left(z_{1}\right), \quad e_{2}=\Gamma\left(z_{2}\right), \\
& z_{1}=\frac{x_{1}}{\rho_{1}}, \quad z_{2}=\frac{x_{2}-\alpha-\gamma \tanh \beta}{\rho_{2}},
\end{aligned}
$$

where $\Gamma(z)=\ln \frac{1+z}{1-z}, \alpha$ is the virtual control, $\gamma$ is a positive constant, and $\beta$ is an auxiliary variable; $\rho_{1}$ and $\rho_{2}$ are prescribed performance functions defined by

$$
\begin{aligned}
& \rho_{1}=\left(\rho_{1,0}-\rho_{1, \infty}\right) e^{-a_{1} t}+\rho_{1, \infty}, \\
& \rho_{2}=\left(\rho_{2,0}-\rho_{2, \infty}\right) e^{-a_{2} t}+\rho_{2, \infty},
\end{aligned}
$$

where $\rho_{1,0}$ and $\rho_{2,0}$ are initial values satisfying $\rho_{1,0}>\left|x_{1}(0)\right|$ and $\rho_{2,0}>\left|x_{2}(0)-\alpha(0)-\gamma \tanh \beta(0)\right|$, respectively, $\rho_{1, \infty}$ and $\rho_{2, \infty}$ are final values that can adjust the region of the steadystate errors; $a_{1}$ and $a_{2}$ are positive constants that can adjust the convergence rate of the errors.

Remark 2. If $e_{1}$ and $e_{2}$ are bounded, $z_{1}$ and $z_{2}$ satisfy $\left|z_{1}\right|<1$ and $\left|z_{2}\right|<1$, respectively. This means that $\left|x_{1}\right|<\rho_{1}$. Since $\rho_{1}$ is selected properly, the angle $x_{1}$ can be stabilized. Therefore, the controller is designed to guarantee the boundedness of $e_{1}$ and $e_{2}$.

Step 1: Using (5), the time derivative of $e_{1}$ is

$$
\dot{e}_{1}=\frac{2}{\rho_{1}} \cosh ^{2} \frac{e_{1}}{2}\left(z_{2} \rho_{2}+\alpha-z_{1} \dot{\rho}_{1}+\gamma \tanh \beta\right) .
$$

By the definition of $\Gamma(\cdot), z_{i}=\tanh \frac{e_{i}}{2}$ where $i=1,2$. Thus, $z_{1}$ and $z_{2}$ are bounded. Furthermore, $\rho_{i}$ and its time derivative are bounded by the definition of (6). This implies that there exists a positive constant satisfying

$$
\left|z_{2} \rho_{2}-z_{1} \dot{\rho}_{1}+\gamma \tanh \beta\right| \leq \Phi_{1}
$$

To stabilize $e_{1}$, choose the virtual control $\alpha$ as follows:

$$
\alpha=-k_{1} e_{1},
$$

where $k_{1}$ is a positive constant.

Consider the Lyapunov function candidate

$$
V_{1}=\frac{1}{2} e_{1}^{2}
$$

The time derivative of $(10)$ along with $(7)$ and $(9)$ is

$$
\begin{aligned}
\dot{V}_{1} & =\frac{2}{\rho_{1}} \cosh ^{2} \frac{e_{1}}{2}\left(z_{2} \rho_{2}+\alpha-z_{1} \dot{\rho}_{1}+\gamma \tanh \beta\right) e_{1} \\
& \leq \frac{2}{\rho_{1}} \cosh ^{2} \frac{e_{1}}{2}\left(-k_{1}\left|e_{1}\right|^{2}+\Phi_{1}\left|e_{1}\right|\right)
\end{aligned}
$$

If $\left|e_{1}\right|>\frac{\Phi_{1}}{k_{1}}, \dot{V}_{1}$ is negative. Thus, $e_{1}$ is bounded. This implies that $\alpha$ and $\dot{\alpha}$ are bounded from (7) and (9).

Step 2: Using (5), the time derivative of $e_{2}$ is

$$
\begin{gathered}
\dot{e}_{2}=\frac{2}{\rho_{2}} \cosh ^{2} \frac{e_{2}}{2}\left(f\left(x_{1}\right)+b\left(x_{1}\right) \bar{u}+d(t)-\dot{\alpha}\right. \\
\left.-z_{2} \dot{\rho}_{2}-\gamma \frac{\dot{\beta}}{\cosh ^{2} \beta}\right) .
\end{gathered}
$$

For the update of the auxiliary variable $\beta$, choose $\dot{\beta}$ as follows.

$$
\dot{\beta}=\frac{\cosh ^{2} \beta}{\gamma}\left(-k_{\beta} \tanh \beta+u-\bar{u}\right),
$$

where $k_{\beta}$ is a positive constant.

Remark 3. In (13), if the actual control input $u$ is larger than its limit $u_{M}$, it will affect the update of the auxiliary variable $\beta$ to compensate the exceeded input. Otherwise, $\beta$ is not working. Therefore, the auxiliary variable $\beta$ can solve the input saturation problem.

Substituting 13 into (12) yields

$$
\begin{gathered}
\dot{e}_{2}=\frac{2}{\rho_{1}} \cosh ^{2} \frac{e_{2}}{2}\left(f\left(x_{1}\right)+\left(b\left(x_{1}\right)+1\right) \bar{u}+d(t)-\dot{\alpha}\right. \\
\left.-z_{2} \dot{\rho}_{2}+k_{\beta} \tanh \beta-u\right) .
\end{gathered}
$$

By Assumption 1, $f\left(x_{1}\right), b\left(x_{1}\right)$, and $d(t)$ are bounded. Moreover, the boundedness of $\dot{\alpha}, z_{2}$, and $\dot{\rho}_{2}$ is proved in Step 1. Thus, there exists a positive constant $\Phi_{2}$ satisfying

$$
\begin{aligned}
\mid f\left(x_{1}\right)+ & \left(b\left(x_{1}\right)+1\right) \bar{u}+d(t) \\
& -\dot{\alpha}-z_{2} \dot{\rho}_{2}+k_{\beta} \tanh \beta \mid \leq \Phi_{2} .
\end{aligned}
$$

To stabilize $e_{2}$, choose the actual control $u$ as follows.

$$
u=k_{2} e_{2},
$$

where $k_{2}$ is a positive constant.

Consider the Lyapunov function candidate

$$
V_{2}=\frac{1}{2} e_{2}^{2}
$$




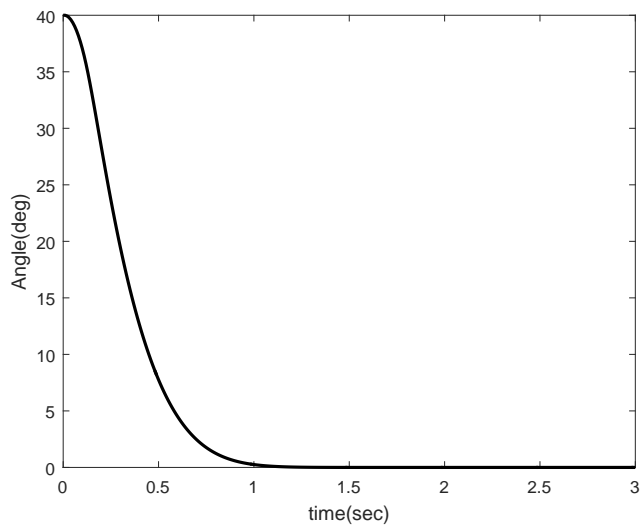

(a)

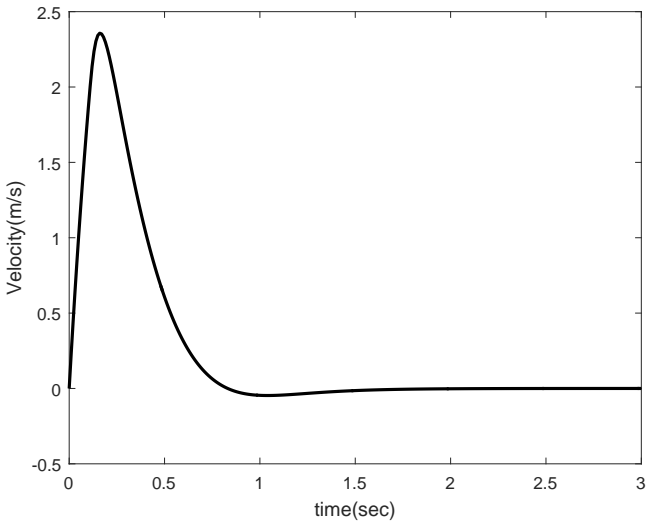

(c)

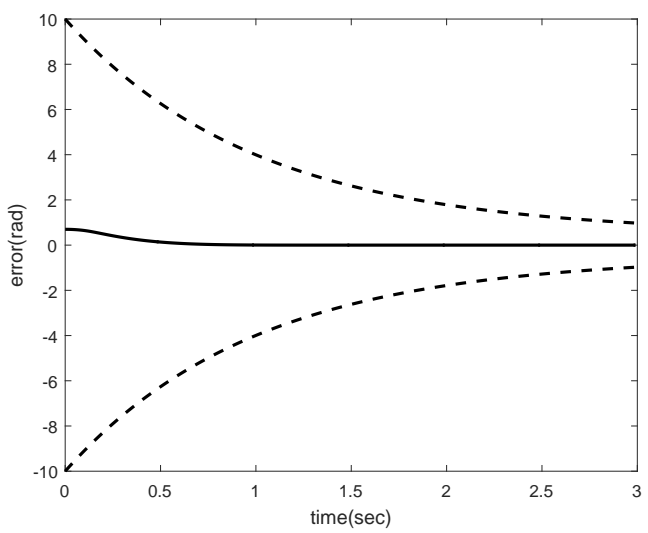

(e)

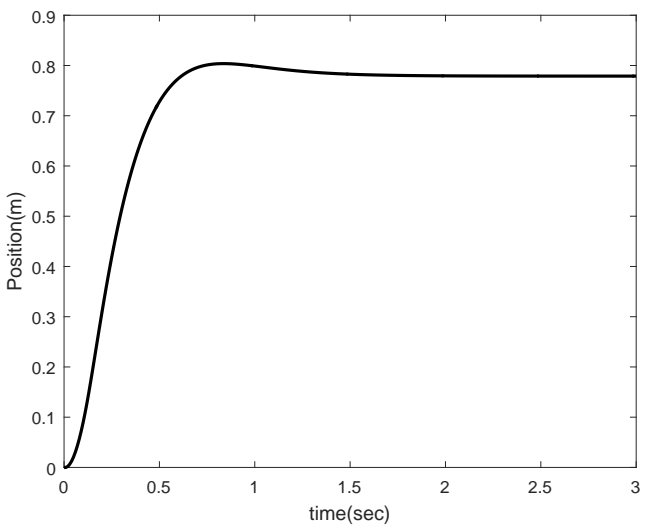

(b)

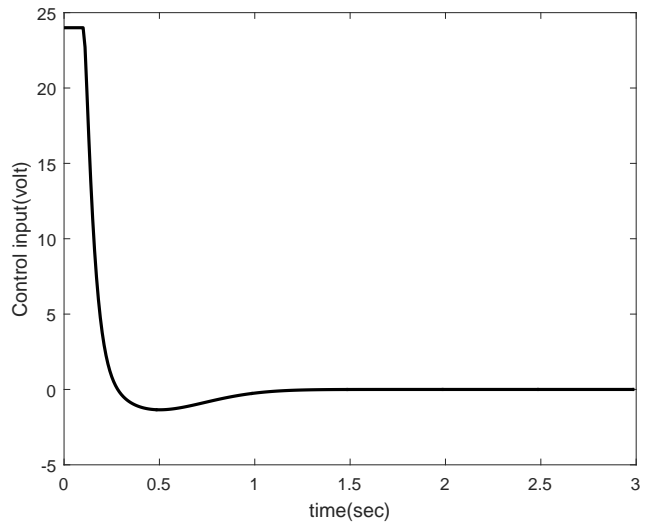

(d)

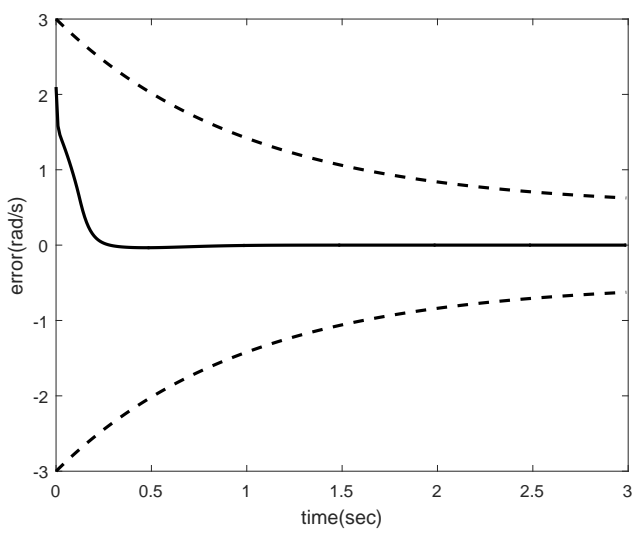

(f)

Figure 2. Response of the Segway with initial condition $\theta(0)=40^{\circ}$ : (a) angle of the inclination; (b) position (c) linear velocity (d) motor input voltage (e) solid : $x_{1}$, dashed : $\rho_{1}$ (f) solid : $x_{2}-\alpha-\gamma \tanh \beta$, dashed : $\rho_{1}$

The time derivative of (17) along with (14) and (16) is

$$
\begin{aligned}
\dot{V}_{2}= & \frac{2}{\rho_{2}} \cosh ^{2} \frac{e_{2}}{2}\left(f\left(x_{1}\right)+\left(b\left(x_{1}\right)+1\right) \bar{u}+d(t)-\dot{\alpha}\right. \\
& \left.-z_{2} \dot{\rho}_{2}+k_{\beta} \tanh \beta-k_{2} e_{2}\right) e_{2} \\
\leq & \frac{2}{\rho_{2}} \cosh ^{2} \frac{e_{2}}{2}\left(-k_{2}\left|e_{2}\right|^{2}+\Phi_{2}\left|e_{2}\right|\right)
\end{aligned}
$$

If $\left|e_{2}\right|>\frac{\Phi_{2}}{k_{2}}, \dot{V}_{2}$ is negative. Thus, $e_{2}$ is bounded.

Theorem 1. Consider the Segway model in (3) with the model uncertainty and input constraint. If the motor input voltage (16) is applied to the Segway, the angle $x_{1}$ remains within the prescribed performance bound. Furthermore, if $\rho_{1, \infty}$ is selected 


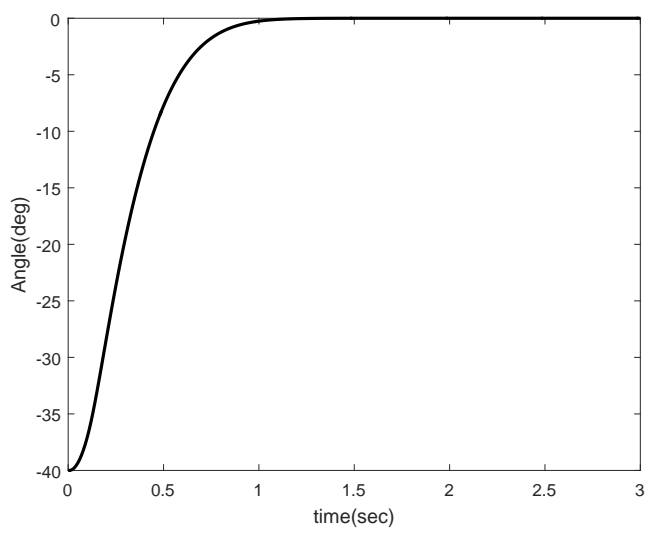

(a)

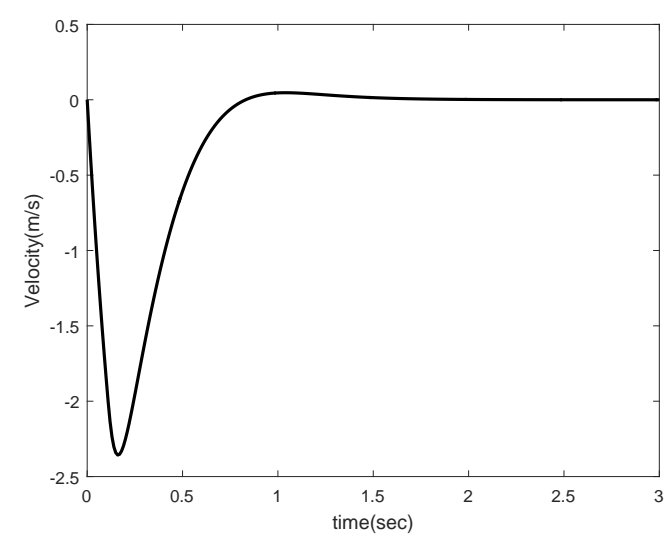

(c)

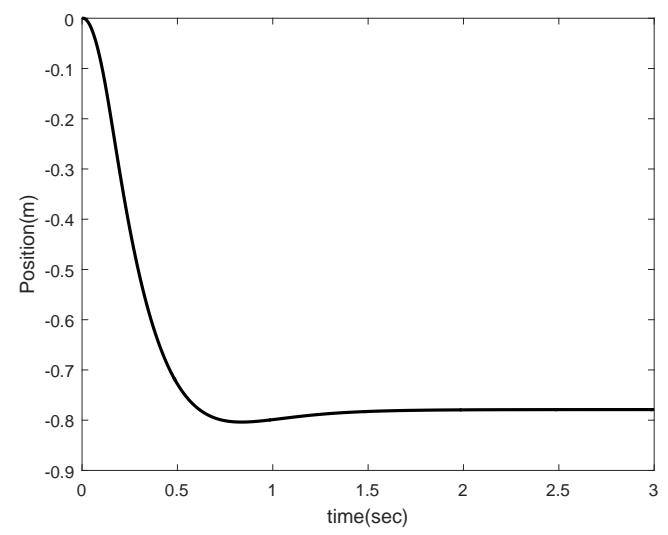

(b)

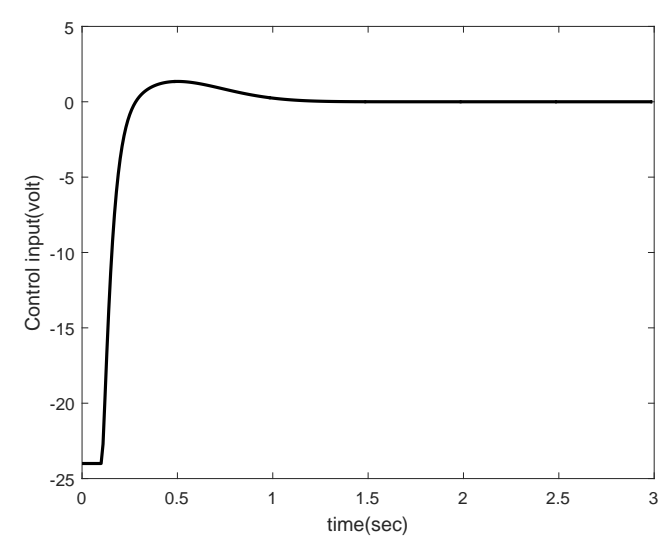

(d)

Figure 3. Response of the Segway with initial condition $\theta(0)=-40^{\circ}$ : (a) angle of the inclination; (b) position (c) linear velocity (d) motor input voltage

near zero, it is moved in the neighborhood of zero.

Proof. From (11) and (18), $e_{1}$ and $e_{2}$ are bounded. This leads $\left|x_{1}\right|<\rho_{1}$ as stated in Remark 1. Thus, $x_{1}$ remains within $\rho_{1, \infty}$ as $t \rightarrow \infty$. This means that if $\rho_{1, \infty}$ is selected near zero, the angle of the inclination is moved in the neighborhood of zero.

\section{Simulation Results}

For real applications, the model parameters in [12] are employed. These variables are shown in Table 1. The control parameters are chosen as $k_{1}=15, k_{2}=40, k_{\beta}=50, \gamma=1$, $\rho_{1,0}=10, \rho_{2,0}=3$, and $\rho_{1, \infty}=\rho_{2, \infty}=0.5$. The limit of the motor input voltage is set to $u_{M}=24 \mathrm{~V}$. To obtain the linear velocity and position of the Segway, Newton's second law is used.
Two different cases, i.e., $\theta(0)=40^{\circ}$ and $\theta(0)=-40^{\circ}$, are considered in the simulation. The simulation results are shown in Figures 2 and 3 . In both cases, the angle of the inclination converges to zero even though the motor control input is saturated. This paper focuses on controlling of the angle of the inclination. Thus, the velocity control is ignored. However, from Figures 2(c) and 3(c), one can know that the linear velocity is also converged to zero as the angle of the inclination goes to zero. This means the stop of Segway if the angle of the inclination is zero. Figures 2 e) and (f) show that the error signals remain within the prescribed performance bounds.

To show the robustness of the proposed scheme against model uncertainties, the simulation is performed with various mass and length. As stated in Section 1, these values are different according to the passenger. The simulation results are shown in Figure 4 One can see that the performance difference for the various model parameters is not significant. 


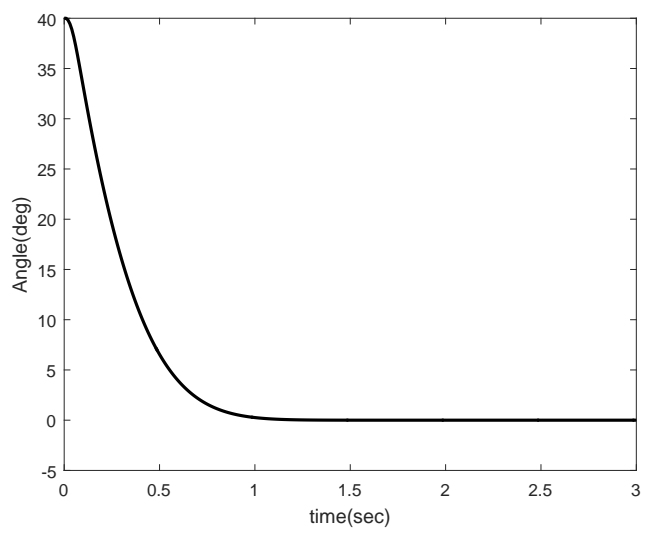

(a)

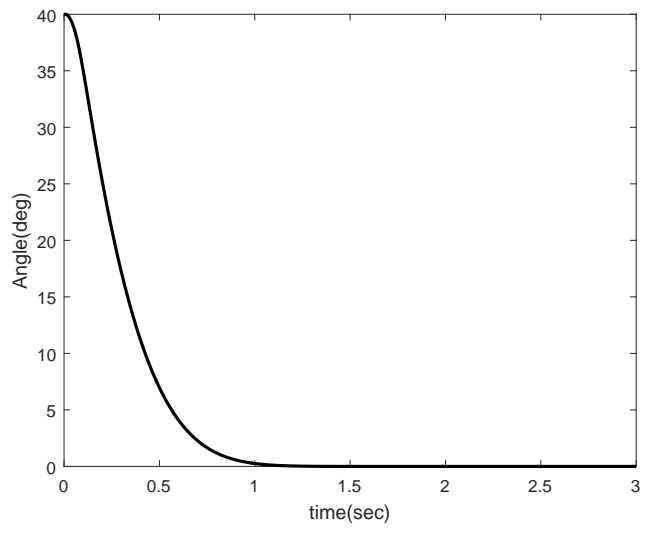

(b)

Figure 4. Response of the Segway: (a) $m=60 \mathrm{~kg}, l=0.85 \mathrm{~m}$; (b) $m=40 \mathrm{~kg}, l=0.8 \mathrm{~m}$

Table 1. Definitions of parameters of the Segway

\begin{tabular}{ccc}
\hline Parameter & Definition & Value \\
\hline$\theta$ & Angle of the inclination & $\mathrm{rad}$ \\
$k_{t}$ & Constant of the motor torque & $0.869 \mathrm{~N} / \mathrm{A}$ \\
$l$ & Length & $0.85 \mathrm{~m}$ \\
$r$ & Wheel radius & $0.2 \mathrm{~m}$ \\
$R$ & Resistance of the motor & $1 \Omega$ \\
$m$ & Mass of the passenger & $85 \mathrm{~kg}$ \\
$M$ & Mass of the Segway & $10 \mathrm{~kg}$ \\
$I$ & Inertia of the Segway & 68.98 \\
& & $\mathrm{~kg} \cdot \mathrm{m}^{2}$ \\
& Acceleration of gravity & $9.8 \mathrm{~m} / \mathrm{s}^{2}$ \\
$n$ & Gear reduction & 10 \\
\hline
\end{tabular}

\section{Conclusions}

In this paper, the tracking control problem of Segway has been investigated. The Segway is a nonlinear system with the model uncertainty and time-varying control coefficient due to various passengers. This requires the robust controller to deal with the model uncertainty and time-varying control coefficient. For this, the controller is designed using the prescribed performance functions and auxiliary variable. With the help of the auxiliary variable, the controller can solve the input saturation problem and does not require the Nussbaum gain technique which is generally used to deal with time-varying control coefficient. This leads the simple controller unlike other nonlinear controllers for Segway. The performance of the proposed scheme is verified by numerical simulations.

\section{Conflict of Interest}

No potential conflict of interest relevant to this article was reported.

\section{Acknowledgment}

This work was supported by the research grant of the Kongju National University in 2015.

\section{References}

[1] C. Yang, Z. Li, R. Cui, and B. Xu, "Neural network-based motion control of an underactuated wheeled inverted pendulum model," IEEE Transactions on Neural Networks and Learning Systems, vol. 25, no. 11, pp. 2004-2016, 2014. http://dx.doi.org/10.1109/TNNLS.2014.2302475

[2] J. Fei and H. Ding, "Adaptive sliding mode control of dynamic system using RBF neural network," Nonlinear Dynamics, vol. 70, no. 2, pp. 1563-1573, 2012. http: //dx.doi.org/10.1007/s11071-012-0556-2

[3] Y. H. Chang, W. S. Chan, and C. W. Chang, "T-S fuzzy model-based adaptive dynamic surface control for ball and beam system," IEEE Transactions on Industrial Electronics, vol. 60, no. 6, pp. 2251-2263, 2013. http://dx.doi.org/10. 1109/TIE.2012.2192891

[4] M. M. Fateh and S. Khorashadizadeh, "Robust control of electrically driven robots by adaptive fuzzy estimation of 
uncertainty," Nonlinear Dynamics, vol. 69, no. 3, pp. 14651477, 2012. http://dx.doi.org/10.1007/s11071-012-0362-x

[5] Q. Xu and M. Jia, "Model reference adaptive control with perturbation estimation for a micropositioning system," IEEE Transactions on Control Systems Technology, vol. 22, no. 1, pp. 352-359, 2014. http://dx.doi.org/10.1109/ TCST.2013.2248061

[6] C. Yang, Z. Li, and J. Li, "Trajectory planning and optimized adaptive control for a class of wheeled inverted pendulum vehicle models," IEEE Transactions on Cybernetics, vol. 43, no. 1, pp. 24-36, 2013. http://dx.doi.org/10. 1109/TSMCB.2012.2198813

[7] A. Boulkroune and M. M'saad, "On the design of observerbased fuzzy adaptive controller for nonlinear systems with unknown control gain sign," Fuzzy Sets and Systems, vol. 201, pp. 71-85, 2012. http://dx.doi.org/10.1016/j.fss.2011. 12.005

[8] S. Tong, S. Sui, and Y. Li, "Adaptive fuzzy decentralized output stabilization for stochastic nonlinear large-scale systems with unknown control directions," IEEE Transactions on Fuzzy Systems, vol. 22, no. 5, pp. 1365-1372, 2014. http://dx.doi.org/10.1109/TFUZZ.2013.2291554

[9] C. P. Bechlioulis and G. A. Rovithakis, "A low-complexity global approximation-free control scheme with prescribed performance for unknown pure feedback systems," Automatica, vol. 50, no. 4, pp. 1217-1226, 2014. http://dx.doi. org/10.1016/j.automatica.2014.02.020
[10] C. P. Bechlioulis and G. A. Rovithakis, "Guaranteeing preselected tracking quality for uncertain strict-feedback systems with deadzone input nonlinearity and disturbances via low-complexity control," Automatica, vol. 54, pp. 135145, 2015. http://dx.doi.org/10.1016/j.automatica.2015.01. 038

[11] L. J. Pinto, D. H. Kim, J. Y. Lee, and C. S. Han, "Development of a Segway robot for an intelligent transport system," in Proceedings of 2012 IEEE/SICE International Symposium on System Integration, Fukuoka, 2012, pp. 710-715. http://dx.doi.org/10.1109/SII.2012.6427308

[12] W. Younis and M. Abdelati, "Design and implementation of an experimental Segway model," in Proceedings of the 2nd Mediterranean Conference on Intelligent Systems and Automation, Zarzis, 2009, pp. 350-354. http://dx.doi.org/ 10.1063/1.3106501

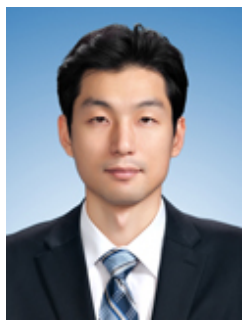

Bong Seok Park received his B.S., M.S., and Ph.D. degrees in Electrical and Electronic Engineering from Yonsei University in 2005, 2008, and 2011, respectively. Since 2015, he has been with the Division of Electrical, Electronic, and Control Engineering, Kongju National University. His research interests include nonlinear control, formation control, and the control of robots 